\title{
The Role of Artificial Intelligence in Many Dental Specialties
}

\author{
Choirunisa Nur Humairo ${ }^{1 *}$, Aquarina Hapsari ${ }^{1}$, and Indra Bramanti ${ }^{2}$ \\ ${ }^{1}$ Residency Students, Department of Pediatric Dentistry, Universitas Gadjah Mada, Yogyakarta, Indonesia \\ ${ }^{2}$ Lecturer, Department of Pediatric Dentistry, Universitas Gadjah Mada, Yogyakarta, Indonesia
}

\begin{abstract}
Background: Technology has become a fundamental part of human living. The evolution of technology has been advantageous to science development, including dentistry. One of the latest technology that draw many attention is Artificial Intelligence (AI). Purpose: The aim of this review is to explain the use of AI in many disciplines of dental specialties and its benefit. Reviews: The application of Artificial Intelligence may be beneficial for all dental specialties, varying from pediatric dentist to oral surgeon. In dental clinic management, AI may assist in medical record as well as other paperwork. AI would also give a valuable contribution in important dental procedures, such as diagnosis and clinical decision making. It helps the dentist deliver the best treatment for the patients. Conclusion: The latest development of Artificial Intelligence is beneficial for dental practitioner in the near future. It is considered as a breakthrough of the $21^{\text {st }}$ century to support the diagnostic procedure and decision making in clinical practice. The use of AI can be applied in most of dental specialties.
\end{abstract}

\section{Introduction}

Technology has become a fundamental part of human living. The evolution of technology has been advantageous to science development, including dentistry. One of the latest technology that draw many attention is Artificial Intelligence (AI). Based on Oxford Learners Dictionary, the definition of Artificial Intelligence is a branch of study particularly focus on making computers replicate the behavior of intelligent human [3]. The Cambridge Academic Content of Dictionary further explained that AI means the utilization of computer programs with special qualities of human intelligence, including picture and speech recognition, as well as experience learning [5]. The aim of this review is to explain the use of AI in various fields of dental specialties and its benefit.

In dentistry, artificial intelligence may help the dentist to diagnose and assist on the clinical decision making in order to give the best treatment for the patients. Moreover, it may investigate if there are any abnormal changes of oral mucosa [6]. Nowadays, intraoral scanners and cameras utilization in dental practice has given a major advantage in the process of diagnosis and treatment planning [1]. The use of Convolutional Neural Network (CNN) will also aid to ease diagnosis procedure during dental practice [7].

Artificial Neural Network (ANN) is a mathematical model or a program in computer that imitate the neural network of the human brain for cognitive role, such as problem solving. A combination of multilayer neural networks may result in deep learning systems, including speech recognition. Deep learning system itself may functionally affect the activities whether they are performed by human or machine $[8,10]$. One of the classes of ANN is Convolutional Neural Network (CNN) which is dominantly in charge for computer vision [9]. Thus $\mathrm{CNN}$ is beneficial to help with the medical imaging. $\mathrm{CNN}$ is able to detect anatomical structures and pathology; separating the structures and the normal findings; and also categorize them into classification. For example, CNN is used to detect dental decay on the exact position of the tooth [7]. It also improves the performance of dental radiology imaging [9]. A research by Lee., et al., (2018) was done to appraise the CNN efficacy to investigate any dental caries on periapical radiographs. There were 3000 periapical radiographic images consisted of premolar, molar and both premolar and molar models. The accuracies of the images were above $80 \%$ for those three categories which showed an impressive result of CNN application in radiology [13].

\section{Discussion}

In general, the use of AI can be applied in dental practice and various number of dental specialties. Below is the explanation and examples of the AI application in the world of dentistry based on each dental specialties.

\subsection{Artificial Intelligence in Pediatric Dentistry}

Behavior modification is an important phase in delivering dental treatments towards pediatric dental patients. The application of AI may give a major change

\footnotetext{
* Corresponding author: choirunisahumairo@mail.ugm.ac.id
} 
for pediatric dentists. Developed technology such as 4D animation and virtual reality games can be an effective behavior modification aid for pediatric dental patients. The use of CAD/CAM technology has been a favorable choice in designing the adult dental patient's smiles, which also can be applied in children concerning aesthetic pleasing treatments. AI based pain control devices may benefit the patient to experience less terrifying dental surgery procedures [14].

\subsection{Artificial intelligence in Restorative Dentistry}

Dental practitioners who have been working for hours may experience fatigue and tiresome, thus they may overlook dental caries during inspection. The use of AI as a software may be highly beneficial to assist the dentist in determining any caries lesion of the teeth on radiograph. A Study by Bhatia (2019) showed that one of intraoral radiographs, which is bitewing, is able to highlight dental caries in the step of early diagnosis. It may increase the awareness of the oral condition of the patients. Therefore, a prevention treatment can be delivered before the caries is getting worse [13].

\subsection{Artificial Intelligence in Orthodontics}

The application of AI in orthodontics has been done by Patkas., et al (2018) to analyze the facial attractiveness and age appearances following orthognatic surgery. The retrospective study was done to 150 patients who have completed orthognatic surgery in Switzerland. The result showed that AI was able to effectively analyze the advantage of orthognathic treatment to increase the patients' attractiveness in $74.7 \%$, mostly post mandibular surgery. The AI technology was also beneficial to assess the age appearances of those patients. [2]

A branch of AI, which is Expert System (ES) has been constructed for an orthodontic research done by Xie., et al. (2010). ES mainly play a part in knowledge and information processing thus it helps with the dentist's decision making as a problem solving process. The study involved participation of 200 subjects aged 11-15 years old in Nanjing, China. The program was able to suggest a decision whether the malocclusion patients need to undergo extraction for orthodontic treatment or not. The result of the study was able to reach $80 \%$ accuracy for the clinical decision making of the dentists' [10].

\subsection{Artificial Intelligence in Periodontics and Surgery}

Artificial intelligence based surgical robot has been developed over the years to imitate both human body and intelligence. The application of AI in surgical robot around the head and neck region has shown a successful procedure, including oral implant, tumour surgery, biopsy and temporomandibular joint operation. A software has been developed for oral surgeons to find out any pain in orofacial region, called RHINOS. Craniofacial surgeon got a major advantage of combining the use of AI and surgical robot because it may shorten the duration of surgery, move gently around delicate internal structure and more precision in conducting the procedure. Furthermore, it may reduce the potentials of revision procedures on resection, such as tumour removal. [20,22].

\subsection{Artificial Intelligence in Prosthodontics}

There are many steps to fulfill before making a dental prosthesis, such as positioning the anatomical facial landmark and calculating the facial measurement. The use of advanced technology would lessen the time spent on the diagnosis procedure and maximize the aesthetic consideration of the patients'. Nowadays, many dental practitioners have gained the benefit of using CAD/CAM application in designing the patient's smile. It provides both $2 \mathrm{D}$ and $3 \mathrm{D}$ image to design and manufacture many dental restoration, including dental crowns and fixed dental prosthesis. A prosthodontic software has also been developed to make a removable partial denture easier, called RaPid. The RaPid software combined the application of $\mathrm{CAD} / \mathrm{CAM}$ as well as computer logic into its program $[17,22,23]$.

\subsection{Artificial Intelligence in Dental Education}

Dental students would experience a very high technology environment during their undergraduate studies. They may practice on virtual patients imitating response of a real patient during preclinical stage instead of working on a phantom [11]. This studying method would reduce the risk of errors regarding the dental treatment procedures on a real patient [12]. Integration of CBCT and MRI with artificial intelligence will ease the radiologist work. [24]

\subsection{Artificial Intelligence in Dentist-Patients Networking}

A good dental clinic is the one provides the best service towards its own patient. This can be so much easier with the use of Artificial Intelligence. It may ease to organize patient's appointments, manage the medical records and insurance. The dental practitioner would be aware of the patient's previous dental and medical condition based on the records. It also would help the dentist in drug prescription following oral surgery. Moreover, it may help the patients as a first aid in dental emergencies before the professional's arrival $[15,16,17,18]$.

\section{Conclusion}

The latest development of Artificial Intelligence is beneficial for dental practitioner in the near future. It is considered as a breakthrough of the $21^{\text {st }}$ 
century to support the diagnostic procedure and decision making in clinical practice. The use of AI can be applied in most of dental specialties.

\section{References}

1. X. Xie, L. Wang, A. Wang, Artificial neural network modelling for deciding if extraction are necessary prior to orthodontic treatment, J. Angle Orthod 80, 262-266 (2010)

2. R. Patcas, D. A. J. Bernini, A. Volokitin, E. Agustsson, R. Rothe, R. Timofte, Applying artificial intelligence to assess the impact of orthognathic treatment on facial attractiveness and estimated age, Intl. J. of Oral Maxillofacial Surg 48, 1, 77-83 (2019) https://doi.org/10.1016/J.IJOM.2018.07.010

3. Information on https://www.oxfordlearnersdictionaries.com/defini tion/english/artificialintelligence? $q=$ artificial + intelligence (2020)

4. C. Brown, Artificial Intelligence in Dentistry, Inside Dent. Tech 7, 10 (2019)

5. Information on https://dictionary.cambridge.org/dictionary/english artificial-intelligence(2020)

6. B. Majumdar, S.C. Saroda, G.S. Saroda, S. Patil, Technology: Artificial Intelligence, B.D.J 224, 9167 (2018).

7. F. Schwendicke, T. Golla, M. Dreher, J. Krois, Convolutional neural networks for dental image diagnostics: a scoping review, J. Dent. 91, 103226,(2019) https://doi.org/10.1016/J.JDENT.2019.103226

8. V. Zwass, Neural network, Encyclopedia Britannica, information on https://www.britannica.com/technology/neuralnetwork (2020)

9. R. Yamashita, M. Nishio, R.K.G. Do, Convolutional neural network : an overview and application in radiology. Insights Imag. 9, 611629 (2018) doi:10.1007/s13244-018-0639-9

10. H. T. Yau, L. S. Tsou \& M. J. Tsai, Octree-based Virtual Dental Training System with a Haptic Device, Computer-Aided Des. App. 3:1-4, 415424, (2006) DOI: $10.1080 / 16864360.2006 .107384$ $\underline{80}$

11. T. Murray, Authoring intelligent tutoring system for visual classification problem solving. Art. Int. in Med 36, 85 - 117 (2006)

12. J.H. Lee, D.H. Kim, S.N. Jeong, S.H. Choi, Detection and diagnosis of dental caries using a deep learning-based convolutional neural network algorithm, J. Dent. 77, 106-111 (2018) https://doi.org/10.1016/j.jdent.2018.07.015.

13. A.P. Bhatia, Artificial intelligence: an advancing front of dentistry. Acta Scientific Dent. Sci. 3, 135- 138 (2019)
14. M.S. Baliga, Artificial intelligence-the next frontier in pediatric dentistry, J.Indian.Soc.Pedod.Prev.Dent 37, 315 (2019)

15. S.S. Khanna, P.A. Dhaimade, Artificial intelligence: transforming dentistry today. Indian J. Basic Applied Med. Res. 6, 161 - 167 (2017)

16. B. Majumdar, Technology: artificial intelligence, B.D.J 224, 916 - 917 (2018)

17. B. Alexander, J. Sunil, Artificial intelligence in dentistry current concepts and a peep into the future, Intl J. Advanced Res. 6,12, 1105-1108 (2018)

18. D.P. Saemant, Stereolithhographic surgical templates for placement of dental implants in complaxes cases. Intl J. Periodontics and Restorative Denti. 23.3, 287 -295 (2003)

19. M.S. Baliga, Artifcial intelligence - The next frontier in pediatric dentistry, J Indian Soc Pedod Prev Dent 37, 315 (2019)

20. P. Hammond, J.C. Davenport, F.J. Fitzpatrick, Logic based constraints and design of dental prothesis, Art. Int. in Med 5, 431-446 (1993)

21. I. Susic, M. Travar, M. Susic, The application of cad/cam technology in dentistry, IOP Conf. Ser.: Mater. Sci. Eng. 200, 012020 (2017)

22. E.A. Medonce, Clinical decision support systems: perspectives in dentistry, J. Dent. Ed. 68, 589-597 (2014)

23. J.J. Hwang, An overview of deep learning in the field of dentistry. Imaging Science in Dentistry. 49, 1-7 (2019)

24. J. Bradley, Human-computer interaction and the growing role of social context. Bul. Am. Soc. Info. Sci. Tech. 24, 18-19 (1998) doi:10.1002/bult.85 